E. Börger G. Jäger H. Kleine Büning

M. M. Richter (Eds.)

\title{
Computer Science Logic
}

5th Workshop, CSL ' 91

Berne, Switzerland, October 7-11, 1991

Proceedings

Springer-Verlag

Berlin Heidelberg New York

London Paris Tokyo

Hong Kong Barcelona

Budapest 


\section{Table of Contents}

The expressive power of transitive closure and 2-way multihead automata

Y. Bargury and J. Makowsky . . . . . . . . . . . . . . . . . . . . . . . 1

Correctness proof for the WAM with types

Ch. Beierle and E. Börger

Model checking of persistent Petri nets

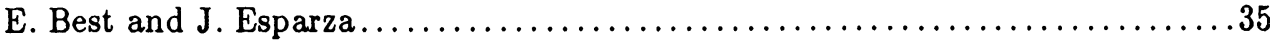

Provability in TBLL: $A$ decision procedure

J. Chirimar and J. Lipton

How to implement first order formulas in local memory machine models

E. Dahlhaus

A new approach to abstract data types II. Computation on ADTs as ordinary computation

S. Feferman.

A primitive recursive set theory and $A F A$ : On the logical complexity

of the largest bisimulation

T. Fernando...

On bounded theories

J. Flum

The cutting plane proof system with bounded degree of falsity

A. Goerdt

Denotational versus declarative semantics for functional programming

J. C. González Moreno, M. T. Hortalá González and M. Rodríguez Artalejo....134

On transitive closure logic

E. Grädel.

Some aspects of the probabilistic behavior of variants of resolution

P. Heusch and E. Speckenmeyer.

Safe queries in relational databases with functions

J. Hirshfeld.

Logical inference and polyhedral projection

J. N. Hooker .

Stable logic

B. Hösli

A transformational methodology for proving termination of logic programs

M. R. K. Krishna Rao, D. Kapur and R. K. Shyamasundar

Plausibility logic

D. Lehmann 
Towards Kleene algebra with recursion

H. Leiß

Equational specification of abstract types and combinators

K. Meinke

Normal forms in infinite-valued logic: The case of one variable

D. Mundici...

A fragment of first order logic adequate for observation equivalence

H. Oḡuztüzün

Ordinal processes in comparative concurrency semantics

S. Pinchinat

Logical semantics of modularisation

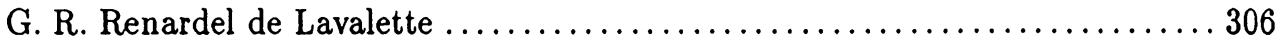

A cut-elimination procedure designed for evaluating proofs as programs

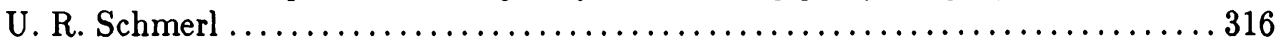

Minimal from classical proofs

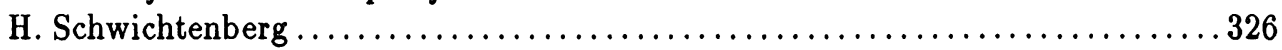

Quantifier hierarchies over word relations

S. Seibert.

Complexity results for the default-and the autoepistemic logic

E. Steffen

On completeness for NP via projection translations

I. A. Stewart ...

Control of $\omega$-automata, Church's problem, and the emptiness problem for tree $\omega$-automata

J. G. Thistle and W. M. Wonham

Comparing the theory of representations and constructive mathematics

A. S. Troelstra

Infinitary queries and their asymptotic probabilities I: Properties definable in transitive closure logic

J. Tyszkiewicz

On completeness of program synthesis systems

A. Voronkov

Proving termination for term rewriting systems

A. Weiermann

This article was processed using the $\mathrm{ITT}_{\mathrm{E}} \mathrm{X}$ macro package with LLNCS style 


\title{
Minimal from Classical Proofs
}

\author{
Helmut Schwichtenberg \\ Mathematisches Institut, Universität München \\ Theresienstr. 39, D-8000 München 2
}

We consider the $\rightarrow \forall$-fragment of first order logic with a distinguished predicate symbol $\perp$ (for falsity); as usual we write $\neg \varphi$ for $\varphi \rightarrow \perp$. Gentzen's natural deduction system for minimal logic in this language consists just of introduction and elimination rules for $\rightarrow$ and $\forall$. Hence any proof in this system gives rise to a typefree $\lambda$-term, possibly with assumption variables. If in addition a proof (and hence also its associated $\lambda$-term) is normal, then from its context, i.e. the assignment of assumption formulas to its assumption variables, and from its endformula we can recover all formulas in the proof. This representation of formal proofs seems to be useful: for instance it allows an efficient implementation of normalization by evaluation (cf. [1], [2]).

It is well known that any proof can be transformed into a unique normal form with respect to $\beta$-conversion. Using $\eta$-expansion we can then construct the long normal form, where all minimal formulas are atomic.

We are interested in the problem of how to find proofs in minimal logic, from a somewhat practical point of view.* In particular we want to make use of existing theorem provers based on classical logic. So our problem is to review under what circumstances a classical proof can be converted into a proof in minimal logic, and moreover to describe reasonable algorithms which do this conversion. A good survey of the subject can be found in [3, Chapter 2.3]. Here we add a new result.

Note first that a convenient way to represent classical logic in our setting is to add stability assumptions of the form

$$
\operatorname{stab}_{P}: \forall \vec{x} \cdot \neg \neg P \vec{x} \rightarrow P \vec{x}
$$

for all predicate symbols $P$. For then we can easily derive $\neg \neg \varphi \rightarrow \varphi$ for an arbitrary formula $\varphi$, using

$$
\begin{gathered}
(\neg \neg \psi \rightarrow \psi) \rightarrow \neg \neg(\varphi \rightarrow \psi) \rightarrow \varphi \rightarrow \psi \\
(\forall x . \neg \neg \varphi \rightarrow \varphi) \rightarrow \neg \neg \forall x \varphi \rightarrow \forall x \varphi,
\end{gathered}
$$

which are derivable in our $\rightarrow \forall$-fragment of minimal logic. Hence by a classical proof of $\psi$ from assumptions $\varphi_{1}, \ldots, \varphi_{n}$ we mean a proof in minimal logic using stability assumptions in addition to the given assumptions $\varphi_{1}, \ldots, \varphi_{n}$.

A formula is called Horn formula if it has the form $\forall x_{1}, \ldots, x_{n} . A_{1} \rightarrow \ldots \rightarrow$ $A_{m} \rightarrow B$ with $A_{i}$ and $B$ atomic. It is called definite Horn formula if in addition we have $B \neq \perp$. If instead of atomic $A_{i}$ we allow universally quantified atomic formulas, the result is called a generalized (definite) Horn formula.

* At the conference I gave a more general lecture on "Proofs and Programs". Since most of what I have said is already published (in [1] and [2]), this note only elaborates one part of the lecture dealing with a very special aspect of the field. 
Theorem 1. Let $\varphi_{1}, \ldots, \varphi_{n}$ be generalized Horn formulas. We have a quadratic algorithm transforming a classical proof in long normal form of $\perp$ from $\varphi_{1}, \ldots, \varphi_{n}$ into a proof in minimal logic of $\perp$ from the same assumptions.

The proof is by induction on the total number of stability axioms used. Note first that bound assumption variables $u$ in the given normal proof can only occur in the context

$$
\operatorname{stab}_{P} \vec{r}(\lambda u d)
$$

with $u$ of type $\neg P \vec{r}$ and $d$ of type $\perp$. The reason for this is that all top formulas different from stability axioms are generalized Horn formulas which never have an implication in the premise of another implication.

Case 1. There is at least one occurrence of a bound assumption variable in the proof. Since we assume our proof to be in long normal form, any of the occurrences of an assumption variable $u$ of type $\neg P \vec{r}$ must be the main premise of an $\rightarrow$-elimination, i.e. must be in a context $u d_{1}$ where $u$ derives $P \vec{r}$. Now choose an uppermost occurrence of a bound assumption variable, i.e. a subderivation $u d_{1}$ where $d_{1}$ does not contain an occurrence of any bound assumption variable. Since $d_{1}$ derives $P \vec{r}$, we can replace the whole subderivation $\operatorname{stab}_{P} \vec{r}(\lambda u d)$ of $P \vec{r}$ (the one where $u$ is bound) by $d_{1}$. Hence we have removed one occurrence of a stability axiom.

Case 2. Otherwise. If there are no more stability axioms in the proof, we are done. If not, choose an uppermost occurrence of a stability axiom, i.e. a subderivation $\operatorname{stab}_{P} \vec{r}(\lambda u d)$ where $d$ does not contain stability axioms. Since we are in case 2 here $d$ also cannot contain free assumption variables which are bound elsewhere in the proof. But since $d$ derives $\perp$, we can replace the whole proof (which also has $\perp$ as its end formula) by $d$ and hence we are done again.

Note that Theorem 1 is best possible in the sense that it becomes false if we allow an implication in the body of one of the Horn formulas. A counterexample (due to U. Berger) is

$$
((P \rightarrow Q) \rightarrow \perp) \rightarrow(P \rightarrow \perp) \rightarrow \perp,
$$

which is provable in classical but not in minimal logic. For if it were, we could replace $\perp$ in this proof (which in minimal logic is just another propositional variable) by $P$, and hence we would obtain a proof in minimal logic of the Peirce formula

$$
((P \rightarrow Q) \rightarrow P) \rightarrow P,
$$

which is known to be underivable.

By essentially the same argument we obtain the following variant of Theorem 1 for generalized definite Horn formulas:

Theorem 2. Let $\varphi_{1}, \ldots, \varphi_{n}$ be generalized definite Horn formulas. We have a quadratic algorithm transforming a classical proof in long normal form of an atomic formula $B$ from $\varphi_{1}, \ldots, \varphi_{n}$ into a proof in minimal logic of $B$ from the same assumptions.

The proof is by a simple modification of the argument for Theorem 1 . Note that in case 2 it cannot happen that stability axioms occur in the proof since then we would have a derivation $d$ of $\perp$ from definite Horn formulas, which is clearly impossible. 


\section{References}

1. U. Berger, H. Schwichtenberg: An inverse of the evaluation functional for typed lambda calculus. Proc. 6th IEEE Symp. on Logic in Computer Science, (ed.: R. Vemuri), Los Alamitos: IEEE Computer Society Press, pp. 203-211, 1991

2. H. Schwichtenberg: Proofs as Programs. To appear in: Leeds Proof Theory ' 90 (ed.: P. Aczel, H. Simmons, S.S. Wainer), Cambridge: University Press, 1992

3. A. Troelstra, D. van Dalen: Constructivism in Mathematics. Vol. I, Amsterdarn: North-Holland, 1988 